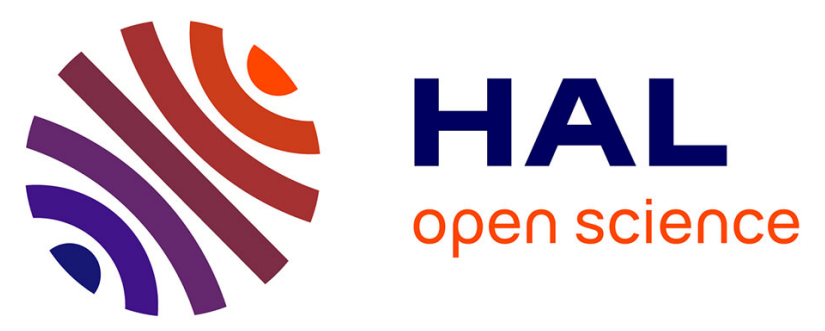

\title{
Improving Health Care Workers' Compliance with Traceability by Recording the Nursing Process at the Point of Care Using a Personal Digital Assistant with a Barcode
}

Olga Florea, Jean-Charles Dufour, Chloe Magnin, Philippe Brouqui, Sophia Boudjema

\section{To cite this version:}

Olga Florea, Jean-Charles Dufour, Chloe Magnin, Philippe Brouqui, Sophia Boudjema. Improving Health Care Workers' Compliance with Traceability by Recording the Nursing Process at the Point of Care Using a Personal Digital Assistant with a Barcode. Journal of Nursing \& Care, 2020, 9, pp.3. 10.37421/jnc.2020.9.500 . hal-02937985

\section{HAL Id: hal-02937985 \\ https://hal-amu.archives-ouvertes.fr/hal-02937985}

Submitted on 28 Sep 2020

HAL is a multi-disciplinary open access archive for the deposit and dissemination of scientific research documents, whether they are published or not. The documents may come from teaching and research institutions in France or abroad, or from public or private research centers.
L'archive ouverte pluridisciplinaire HAL, est destinée au dépôt et à la diffusion de documents scientifiques de niveau recherche, publiés ou non, émanant des établissements d'enseignement et de recherche français ou étrangers, des laboratoires publics ou privés. 


\title{
Improving Health Care Workers'Compliance with Traceability by Recording the Nursing Process at the Point of Care Using a Personal Digital Assistant with a Barcode
}

\author{
Olga Florea ${ }^{1,2}$, Jean Charles Dufour ${ }^{3}$, Chloe Magnin ${ }^{1,3}$, Philippe Brouqui ${ }^{1,2}$ and Sophia Boudjema ${ }^{1,2 *}$ \\ ${ }^{1}$ Aix Marseille Université, IRD, MEPHI, IHU-Méditerranée Infection, Marseille, France \\ ${ }^{2} \mathrm{AP}$-HM, IHU-Méditerranée Infection, Marseille, France \\ ${ }^{3}$ Aix Marseille Université, AP-HM, INSERM, IRD, SESSTIM, Hop Timone, BioSTIC, Marseille, France
}

\begin{abstract}
Background: Adverse events are serious, and frequent complications are most often linked to the quality of nursing care.

Purpose: We evaluated the compliance to traceability of bedside nursing care using the Patient Smart Reader ${ }^{\circledR}$, a personal digital assistant with a barcode.

Methods: We compared paper record forms, specific computer software in the hospital information system and the Patient Smart Reader ${ }^{\circledR}$.

Results: The Patient Smart Reader enhanced the recording of $90 \%$ of the nursing care surveyed. Regarding the insertion of blood catheters, compliance rates increased from $44.19 \%$ to $100 \%$, and blood catheter monitoring increased from $29.64 \%$ to $80.74 \%$. Urinary catheter monitoring and insertion recording increased from $10.23 \%$ to $55.43 \%$ and from $16.67 \%$ to $100 \%$, respectively.

Conclusion: Providing caregivers with a nursing record system that uses barcodes at the point of care in real time significantly improved the traceability of nursing care.
\end{abstract}

Keywords: Electronic records $•$ Nursing records $•$ Personal digital assistant $•$ Adverse event $\bullet$ Hospital-acquired infection $\bullet$ Blood catheter $\bullet$ Urinary catheter • Traceability

\section{Introduction}

An adverse event $(\mathrm{AE})$ is any unexpected event that follows any act or action performed or prescribed by a health professional. Adverse events are widespread and have a significant cost; it was estimated that the total costs of preventable AEs in the USA were between $\$ 17.1$ billion and $\$ 29$ billion annually [1]. The two most frequent classes of $A E s$, postoperative infections and pressure ulcers, accounted for the highest annual costs (6.5 billion USD) [2]. The prevalence of AEs can vary from 7 to $40 \%$, but it is important to note that AEs can be avoided in more than half of the cases and that $27.6 \%$ of $A E s$ are related to negligence and $76.8 \%$ to inappropriate nursing care [2,3]. In intensive care units (ICUs), AEs are associated with more extended hospital stays [4]. In Canadian hospitals, a study reported that $20.8 \%$ of deaths are related to $\mathrm{AEs}$, and the authors estimated that the death rate due to $\mathrm{AEs}$ in Canada as a whole is $>38,000$ annually [5]. The expected goal in caring for patients is to provide low mortality, low morbidity, and a low readmission ratio after 30 days, with a better quality of life. The Institute of Medicine suggests that improving data collection and analysis of direct patient care would enhance patient safety [6]. According to the "knowing how to prevent" adage, traceability of care is likely to be a significant component of the surveillance and prevention of AEs, suggesting that better traceability should lower the frequency of

*Address for Correspondence: Sophia Boudjema, IHU Mediterranee Infection, 19-21 BD Jean Moulin, 13005, Marseille, France, Tel: 33621523355, E-mail: sophia.boudjema@univ-amu.fr

Copyright: (c) 2020 Florea 0, et al. This is an open-access article distributed under the terms of the Creative Commons Attribution License, which permits unrestricted use, distribution, and reproduction in any medium, provided the original author and source are credited.
AEs and their consequences. The use of the 'Check List' in anesthesia has demonstrated a significant reduction in mortality [7]. It has been suggested that as many as $70 \%$ of adverse events could be avoided if the right information about the right patient is available at the right time, and health information exchange makes this possible. In agreement with the recommendations of most national authorities, the care provided by healthcare workers (HCWs) is currently registered in the patient's hospital electronic health record (EHR). The quality of nursing care is related to the execution of the nursing process, which should be adequately documented [8]. Currently, transferring from paperbased documentation to electronic documentation is commonly available worldwide. Whether computerized or not, the patient's health information is most often recorded manually and outside of the location of the nursing care (the patient's room), resulting in forgetfulness and inaccuracy. While many studies throughout the world have evaluated the impact of EHRs on the quality of nursing care, few studies have specifically evaluated the pertinence of a bedside care recorder for the quality of traceability. A comparison of personal digital assistant (PDA) records at the bedside with paper formats suggest that PDAs used at the bedside are reliable, allow for fewer errors, are easy to use, and are advocated by interviewers $[9,10]$. To improve the quality of nursing care records, the nursing care act should be recorded in real time, meaning that a bedside solution is needed. Some studies have reported nursing record evaluations at the point of care [11,12], but quantitative analyses of compliance with the traceability of nursing care records have not yet been reported in prospective comparative case-control protocols.

The researchers of this study developed a personal digital assistant with a barcode, named the Patient's Smart Reader (PSR), to trace nursing care at the patient's bedside in real time. The aim of this study is to evaluate the impact of such a tool on the compliance of HCWs with recording nursing care in the care unit by successively comparing different nursing care recording tools. The hypothesis was that recording nursing care at the patient's bedside in real time would improve compliance with the traceability of nursing care. 


\section{Materials and Methods}

This study was conducted in the infectious and tropical diseases medical unit of the university hospital in Marseille, France, which consisted of 17 beds. For the purpose of our research, we decided to divide the unit into two distinct parts, namely, nine beds (for cohort A) and eight beds (for cohort B). All voluntary HCWs (doctors, nurses, nursing assistants, and housekeeping personnel) of the unit were included in the study.

\section{Recorder systems}

When a HCW provides nursing care to a patient, he/she must track the care in the nursing care record. The validity of this information must be certified with the time and date of the nursing care and with the signature of the HCW. We compared different traceability systems that were used in patient medical records. The oldest traceability system was a dedicated paper form. This sheet of paper was placed into the patient's paper-based medical record. The second traceability system, the software Pharma ${ }^{\circledR}$ (version 5.8.70602.1 300), was introduced in our institute in 2013 and allows the traceability of medical prescriptions and administered drugs but also provides some of the patient's parameters, such as urine strip, temperature and weight. The third system, introduced in our institute at the beginning of 2016, was an EHR (Axigate ${ }^{\circledR}$ version: 5.6.1P9) embedded in the Hospital Information System (HIS). This EHR allows the registration of some other parameters, such as inserting and monitoring a peripheral venous catheter, inserting and monitoring a urinary catheter, providing an inpatient booklet, and monitoring isolation. Finally, the last system, introduced in June 2016, was the Patient Smart Reader (PSR) and was installed in 8 patient rooms (Cohort B).

The PSR system is a personal digital assistant with a barcode scanner that allows for bedside recording of nursing care (https://vimeo.com/205512348). With the PSR, the HCW can record, at the patient's bedside and in real time, nursing care such as the insertion and monitoring of urinary or peripheral venous catheters by using barcoded implements, or the HCW can directly record various parameters such as body temperature, blood pressure or pain directly onto the PSR. The PSR provides automatic reminders of controls or alerts. For example, recording the insertion of a peripheral venous catheter automatically triggers monitoring every 8 hours. The information collected is transmitted to a server that ensures data storage and facilitates data analysis. Reports and alerts are generated by the server and displayed on the main screen that is available in the staff room, as well as on the PSR.

\section{Data collection}

First, through a before/after study, we retrospectively compared the compliance of data reported on single sheets of paper from April 2012 to March 2013 (period 1) to that collected in both the Pharma ${ }^{\circledR}$ software and paper sheets from March 2013 to April 2014 (period 2). Then, we compared in a prospective cohort study, from June 2016 to January 2017, the compliance to traceability using our institutional available systems, paper sheets plus Pharma ${ }^{\circledR}$ software and EHR (Cohort A) against the PSR alone (Cohort B). For this purpose, the HCWs were asked to use only the PSR to record care in the eight equipped rooms.

\section{Data analysis}

We used descriptive statistics, and we described data compliance in terms of frequencies and percentages. The compliance to traceability (as defined by variable recorded/variable to be recorded) of nursing care using different tools (paper sheet versus paper sheet and Pharma software or any kind of recording systems versus PSR) was analyzed. The obtained differences in compliance were defined as significant when $p<0.05$ (chi-square test). Similarly, the relative risk and a $95 \%$ confidence interval were reported.

\section{Variables studied}

It was decided to monitor the compliance with traceability of 10 variables selected for their public health impact on the safety of care or their mandatory systematic nature. Some of these acts are mandatory for all patients (welcome inpatient booklet, urine strip, weight once per hospital stay upon patient's admittance, body temperature three times a day, and catheter monitoring when appropriate), but blood cultures, blood and urinary catheter insertion and isolation monitoring were prescribed by a medical doctor.

\section{Inclusion/exclusion criteria}

All patients with a length of stay that was less than 5 days were excluded from the study. The length of at least 5 days was chosen to allow a sufficient amount of time in the hospital setting such that a catheter could be prescribed, and other nursing care could be provided as needed.

\section{Ethics}

Patients were informed of the study upon being admitted to the unit. They were informed that their participation in the study would not affect their medical care. The study was approved by our independent ethics committee under $\mathrm{N}^{\circ}$ 2018-011.

\section{Results}

Medical records for 1532 patients were reviewed, and 732 files were excluded from the study because the length of stay was less than five days. Finally, 800 files were considered suitable for analysis, and 18,455 opportunities were assessed for compliance.

Table 1 summarizes the compliance to traceability of the 10 variables between paper forms (period 1; 407 files) and paper forms plus Pharma software (period 2; 393 files). The paper forms reported a low compliance with traceability; the highest rates were blood catheter monitoring $(59.07 \%)$ and temperature $(58.67 \%)$, followed by weight $(55.28 \%)$, welcome inpatient booklet $(46.93 \%)$, blood culture $(43.66 \%)$, blood catheter insertion $(32.51 \%)$ and isolation monitoring (31\%). Urine strip was traced in only $13.51 \%$ of cases, and urinary catheter monitoring showed a meager compliance ratio $(0.62 \%)$. Following the introduction of the Pharma ${ }^{\circledR}$ software, a significant increase in compliance with the traceability of nursing care records was observed for urinary strips, which increased from $13.51 \%$ to $19.85 \%(p=0.02)$, and temperature, which increased from $58.67 \%$ to $87.72 \%$ ( $p<0.0001)$. Regarding the other variables, no significant changes were observed, except for a slight but significant decrease in compliance with blood catheter monitoring $(59.07 \%$ to $56.31 \%, p=0.014$ ).

In the prospective cohort study, a total of 341 files were collected, 148 records were excluded according to the exclusion criteria, and 193 records were included (101 in the cohort $A$ and 92 in the cohort $B$ ), thus representing 6,073 opportunities for analysis.

The use of PSR alone significantly improved the compliance with traceability of all nursing care records except for urinary catheter insertion.

Among the essential findings, according to relative risk and $95 \%$ confidence interval, blood catheter insertion increased from $44.19 \%$ to $100 \%$ of prescribed insertion, and blood catheter monitoring rose from $29.64 \%$ to $80.74 \%$ of catheters monitored. Two other items improved significantly; body temperature increased from $53.12 \%$ to $80.81 \%$, and isolation monitoring increased from $26.57 \%$ to $43.85 \%$ of prescribed isolation (Table 2).

Since then, the system has been deployed in the unit, allowing continuous and real-time monitoring of nursing files. Two years after the completion of this study, the average compliance with traceability of nursing care records of some mandatory items such as temperature, pulse, blood pressure, and pain was 91.7\% (Week 7 February 2019), suggesting that the changes in the behavior of healthcare workers have been sustained.

\section{Discussion}

Reviews of PDA usage in health care indicate that PDAs are widely used, functional, and useful for documentation. Moreover, PDAs might improve decision making, reduce medical errors, and enhance learning for students and professionals $[13,14]$. 
Table 1. Compliance to traceability of 10 variables between papers forms (Period 1) and paper forms plus Pharma software (Period 2).

\begin{tabular}{|c|c|c|c|c|c|c|c|c|}
\hline \multirow{2}{*}{$\begin{array}{c}\text { Nursing care } \\
\text { Variables }\end{array}$} & \multicolumn{3}{|c|}{ Period 1 - 407 files } & \multicolumn{3}{|c|}{ Period 2 - 393 files } & \multirow[b]{2}{*}{ RRR (95\% Cl) } & \multirow[b]{2}{*}{ p-value } \\
\hline & To record & Recorded & Compliance (\%) & To record & Recorded & Compliance (\%) & & \\
\hline Blood catheter insertion & 243 & 79 & $32.51 \%$ & 227 & 82 & $36.12 \%$ & $1.11(0.77-1.58)$ & 0.4669 (NS) \\
\hline Blood catheter monitoring & 4041 & 2387 & $59.07 \%$ & 3717 & 2093 & $56.31 \%$ & $0.95(0.88-1.02)$ & 0.01484 \\
\hline Urinary catheter insertion & 18 & 3 & $16.67 \%$ & 22 & 5 & $22.73 \%$ & $1.36(0.28-6.49)$ & 0.7089 (NS) \\
\hline $\begin{array}{l}\text { Urinary catheter } \\
\text { monitoring }\end{array}$ & 2434 & 15 & $0.62 \%$ & 2237 & 18 & $0.80 \%$ & $1.30(0.65-2.59)$ & 0.5531 (NS) \\
\hline Blood cultures & 142 & 62 & $43.66 \%$ & 96 & 44 & $45.83 \%$ & $1.04(0.65-1.67)$ & 0.8433 (NS) \\
\hline Temperature & 6105 & 3582 & $58.67 \%$ & 5895 & 5171 & $87.72 \%$ & $1.49(1.41-1.58)$ & $<0.0001$ \\
\hline Urine strip & 407 & 55 & $13.51 \%$ & 393 & 78 & $19.85 \%$ & $1.46(1.01-2.13)$ & 0.02086 \\
\hline Isolation monitoring & 4251 & 1318 & $31.00 \%$ & 3507 & 1037 & $29.57 \%$ & $0.95(0.86-1.04)$ & 0.1792 (NS) \\
\hline Weight & 407 & 225 & $55.28 \%$ & 393 & 239 & $60.81 \%$ & $1.10(0.87-1.38)$ & 0.1302 (NS) \\
\hline Welcome booklet & 407 & 191 & $46.93 \%$ & 393 & 186 & $47.33 \%$ & $1.0(0.78-1.28)$ & 0.9662 (NS) \\
\hline
\end{tabular}

Table 2. Compliance to traceability of 10 variables between any type of record* (Cohort A) and PSR (Cohort B).

\begin{tabular}{|c|c|c|c|c|c|c|c|c|}
\hline \multirow{2}{*}{$\begin{array}{c}\text { Nursing care } \\
\text { Variables }\end{array}$} & \multicolumn{3}{|c|}{ Cohort A - 101 files } & \multicolumn{3}{|c|}{ Cohort B- 92 files } & \multirow{2}{*}{ RRR (95\% Cl) } & \multirow{2}{*}{ p-value } \\
\hline & To trace & Traced & Compliance (\%) & To trace & Traced & Compliance (\%) & & \\
\hline Blood catheter insertion & 43 & 19 & $44.19 \%$ & 31 & 31 & $100.00 \%$ & $2.26(1.08-4.71)$ & $<0.0001$ \\
\hline Blood catheter monitoring & 631 & 187 & $29.64 \%$ & 244 & 197 & $80.74 \%$ & $2.72(2.12-3.49)$ & $<0.0001$ \\
\hline Urinary catheter insertion & 6 & 1 & $16.67 \%$ & 2 & 2 & $100.00 \%$ & $6.0(0.33-107.42)$ & 0.1071 \\
\hline $\begin{array}{l}\text { Urinary catheter } \\
\text { monitoring }\end{array}$ & 88 & 9 & $10.23 \%$ & 92 & 51 & $55.43 \%$ & $5.42(2.51-11.66)$ & $<0.0001$ \\
\hline Blood cultures & 63 & 16 & $25.40 \%$ & 26 & 20 & $76.92 \%$ & $3.02(1.36-6.74)$ & $<0.0001$ \\
\hline Temperature & 1508 & 801 & $53.12 \%$ & 1188 & 960 & $80.81 \%$ & $1.52(1.34-1.71)$ & $<0.0001$ \\
\hline Urine strip & 101 & 12 & $11.88 \%$ & 92 & 40 & $43.48 \%$ & $3.65(1.80-7.40)$ & $<0.0001$ \\
\hline Isolation monitoring & 764 & 203 & $26.57 \%$ & 358 & 157 & $43.85 \%$ & $1.65(1.29-2.1)$ & $<0.0001$ \\
\hline Weight & 101 & 29 & $28.71 \%$ & 92 & 69 & $75.00 \%$ & $2.61(1.55-4.38)$ & $<0.0001$ \\
\hline Welcome booklet & 101 & 27 & $26.73 \%$ & 92 & 68 & $73.91 \%$ & $2.76(1.63-4.68)$ & $<0.0001$ \\
\hline
\end{tabular}

${ }^{*}$ Any type of record: variables recorded on any of the available paper forms plus Pharma ${ }^{\circledR}$ software and electronic medical record

A nursing record system allows the care that has been planned or provided to individual patients by nurses or other caregivers to be recorded. Several studies have already compared paper-based and electronic computerized nursing records and suggest that electronic systems are preferred $[15,16]$. Nursing record systems may be an effective way of influencing nurse practice. However, with the use of these technologies, nurses are expected to change the way they document patient care by shifting from paper forms to electronic systems. While the documentation of electronic medical records in the presence of the patient likely impacts the nurse-patient relationship [17], in our experience, bedside recording with a barcode allows for nurses and patients to maintain this relationship. Wang et al. reported that the overall quality of the content documented for the nursing process was not better in the electronic system than in the paper-based system [18]. Interestingly, in our study, the addition of a new nursing record electronic system such as Pharma ${ }^{\circledR}$ improved the traceability of body temperature and urine strip recording, but inversely, the new system significantly decreased compliance with blood catheter monitoring. However, in our study, the lack of significant improvement in urinary catheter insertion traceability with the PSR compared to any type of records reported in Table 2 is likely related to the study power.

To avoid information transmission oversights and improve compliance with the traceability of nursing care, it has been suggested that the recording of care should be performed during bedside care. Moreover, the fight against cross-transmission of pathogens in hospitals, resulting in hospital-acquired infection, explains the choice of a dedicated PDA for each bedroom. Among the weaknesses of our study, randomization was not feasible because the health care organization did not allow a random assignment of patients.

To the best of our knowledge, there is no other study reporting the quantitative evaluation of the point of care for nursing records. The advantage of recording at the point of care is twofold. First, recording at the point of care is safer from a hygiene point of view (one PDA per room). Second, recording at the point of care avoids the loss of information between the patient's bedroom and the nearest computer. Moreover, the data collected by the PSR can be directly transferred to the care plan in the patient's electronic files of the hospital information system. We evaluated ten variables because of their mandatory character, but the PSR contains up to 80 variables and allows for decision-making. For instance, once a nurse has registered the introduction of a peripheral venous catheter, the system will ask her if the catheter should be removed during each shift (8 hours).

\section{Conclusion}

Post-operative infections, such as central and peripheral venous catheterrelated blood stream infections, represent more than half of all AE-related costs. For this reason, we focused our study on the variables associated mostly with hospital-acquired infections rather than drug prescriptions or falls, which are two of the other most frequent AEs reported in the literature. Unfortunately, as the research question focused on the compliance of traceability of nursing care, the adverse events were not recorded. In conclusion, providing a nursing record system to caregivers using barcodes (PSR) at the point of care in real time significantly improved the traceability of nursing care.

\section{Conflicts of Interest}

P Brouqui is one of the founders of the Medihandtrace $\AA$ SAS Startup that owns the copyright of the PSR software.

\section{Acknowledgment}

The authors thank the healthcare personnel of the medical unit for their commitment and participation in this study. This manuscript was edited in English by AJE under ID N4TL8Q9MG. 


\section{Authors' Contributions}

Olga Florea designed the study and wrote the paper. S Boudjema, P Brouqui, and JC Dufour wrote the protocol. C Magnin performed the data analysis. JC Dufour contributed his expertise in medical informatics. JC Dufour, S Boudjema and $\mathrm{P}$ Brouqui revised the manuscript.

\section{Funding}

This study was funded in part by ANR-15-CE36-0004-01 entitled MHT and ANR, IHU Mediterranée Infection 10-IAHU-03.

\section{References}

1. De Vries, EN, Manai Ramrattan, SM Smorenburg and DJ Gouma, et al. "The incidence and nature of in-hospital adverse events: A systematic review." Qual Saf Health Care 17 (2008): v216-223.

2. Schwendimann, Rene, Catherine Blatter, Suzanne Dhaini and Michael Simon, et al. "The occurrence, types, consequences and preventability of in-hospital adverse events: A scoping review." BMC Health Serv Res 18 (2018): 521.

3. D'Amour, Danielle, Carl-Ardy Dubois, Eric Tchouaket and Sean P Clarke, et al. "The occurrence of adverse events potentially attributable to nursing care in medical units: Cross sectional record review." Int J Nurs Stud 51 (2014): 882-891.

4. Ahmed, Adil H, Jyothsna Giri, Rahul Kashyap and Balwinder Singh, et al. "Outcome of adverse events and medical errors in the intensive care unit: A systematic review and meta-analysis." Am J Med Qual 30 (2015): 23-30.

5. Baker, G Ross, Peter G Norton, Virginia Flintoft and Regis Blais, et al. "The Canadian adverse events study: The incidence of adverse events among hospital patients in Canada." CMAJ 170 (2004): 1678-1686.

6. Kohn LT, Corrigan JM and Donaldson MS. To Err is Human: Building a safer health system. Washington DC: National Academic Press, USA, (2000).

7. de Vries, Eefie N, Hubert A Prins, Rogier MPH Crolla and Adriaan J den Outer, et al. "Effect of a comprehensive surgical safety system on patient outcomes." $N$ Engl J Med 363 (2010): 1928-1937.

8. Da Costa Cintia and Graciele Fernanda da Costa Linch. "Implementation of electronic records related to nursing diagnoses." Int J Nurs Knowl (2018).

9. Seebregts Christopher J, Merrick Zwarenstein, Catherine Mathews and Lara Fairall, et al. "Handheld computers for survey and trial data collection in resource-poor settings: Development and evaluation of PDACT, a Palm Pilot interviewing system." Int J Med Inform 78 (2009): 721-731.

10. Zalon, Margarete L, Sonia Sandhaus, Donna Valenti and Ulyana Arzamasova. "Using PDAs to detect cognitive change in the hospitalized elderly patient." Appl Nurs Res 23 (2010): e21-e27.

11. Horng Steven, Foster R Goss, Richard S Chen and Larry A Nathanson. "Prospective pilot study of a tablet computer in an Emergency Department." Int J Med Inform 81 (2012): 314-319.

12. Caligtan Christine A, Carroll Diane L, Hurley Ann C and Gersh-Zaremski Ronna, et al. "Bedside information technology to support patient-centered care." Int J Med Inform 81 (2012): 442-451.

13. Hudson Kathleen and Virginia Buell. "Empowering a safer practice: PDAs are integral tools for nursing and health care." J Nurs Manag 19 (2011): 400-406.

14. Mickan Sharon, Julie K Tilson, Helen Atherton and Nia Wyn Roberts, et al. "Evidence of effectiveness of health care professionals using handheld computers: A scoping review of systematic reviews." J Med Internet Res 15 (2013): e212.

15. Adereti Chinma Stella and Olaogun Adenike Ayobola. "Use of electronic and paperbased standardized nursing care plans to improve Nurses' documentation quality in a Nigerian teaching hospital." Int J Nurs Knowl 30 (2019): 219-227.

16. Urquhart Christine, Rosemary Currell, Maria J Grant and Nicholas R Hardiker. "Nursing record systems: Effects on nursing practice and healthcare outcomes." Cochrane Database Syst Rev 1 (2009): CD002099.

17. Misto Kara, Cynthia Padula, Elizabeth Bryand and Kate Nadeau. "Nurses' perception of the impact of electronic documentation on the Nurse-Patient relationship." J Nurs Care Qual 34 (2019): 163-168.

18. Wang Ning, Ping Yu and David Hailey. "The quality of paper-based versus electronic nursing care plan in Australian aged care homes: A documentation audit study." Int J Med Inform 84 (2015): 561-569.

How to cite this article: Olga Florea, Jean Charles Dufour, Chloe Magnin, Philippe Brouqui, et. al. "Improving Health Care Workers' Compliance with Traceability by Recording the Nursing Process at the Point of Care Using a Personal Digital Assistant with a Barcode." J Nurs Care, Volume 9 (2020):500 doi: $10.37421 /$ jnc.2020.9.500 